\title{
Desarrollando agentes EBDI
}

\author{
Yanet Sánchez, Eva Cerezo \\ GIGA AffectiveLab \\ Instituto de Investigación en Ingeniería de Aragón (I3A) \\ Universidad de Zaragoza, Mariano Esquillor s/n, 50018, Zaragoza, Spain. \\ Tel. +34-976762707, e-mail: ysanchezl@unizar.es
}

\section{Resumen}

En este trabajo se presenta el framework $\mathrm{ABC}$ EDBI para el modelado afectivo de agentes inteligentes con comorptamiento BDI (Belief, Desire, Intention). El mismo integra afecto (emociones, estado de ánimo y personalidad) y capacidades afectivas (regulación del afecto), teniendo en cuenta la influencia de todos los aspectos afectivos en el proceso cognitivo del agente.

\section{Introducción}

Los Agentes Inteligentes, también conocidos como Agentes Autónomos o Agentes Artificiales, se han desarrollado mucho en los ultimos años. Los agentes inteligentes más desarrollados son los agentes corpóreos los cuales se caracterizan por tener un cuerpo que puede ser virtual o físico. Este tipo de agentes presentan capacidades cognitivas como la percepción, el razonamiento, la comunicación, el planeamiento, el aprendizaje y la toma de decisiones. De ambos tipos el más desarrollado son los ECA (Embodied conversational Agent) [1], que son agentes virtuales corpóreos capaces de interactuar cara a cara con el usuario incluyendo rasgos verbales y no verbales (gestos, expresiones faciales, postura corporal, ect.), convirtiéndose en una herramienta poderosa para la disciplina de interacción hombrecomputadora con un ámbito de aplicación muy variado como guías de museo [2], con fines pedagógicos [3], como pacientes virtuales [4], en virtual storytelling [5], en la robótica [6], etc.

Para dar soporte a los agentes inteligentes se han desarrollado arquitecturas cognitivas y frameworks cognitivos. De ambos, los más desarollados son los framework cognitivos, siendo el BDI (Belief Desire Intention) [7] el más difundido y utilizado en el modelado cognitivo de los agentes inteligentes. Este último, tiene un fuerte basamento filosófico y se ha convertido en una herramienta muy popular para el modelado de agentes virtuales. Los agentes inteligentes implementados con este framework se les conoce como agentes BDI y su éxito radica en su sencillez para implementar el proceso de razonamiento lógico de un agente inteligente, el cual está basado en tres aptitudes mentales fundamentales: creencias, deseos e intenciones. Las creencias representan la información que tiene el agente del entorno y de si mismo, los deseos representan el estado motivacional del agente y son los objetivos que desea alcanzar el agente y las intenciones son el conjunto de acciones o planes que se llevan a cabo para cumplir o archivar los deseos trazados y que además proveen el carácter deliberativo al modelo.

Actualmente el desarrollo de los agentes BDI está encaminado a incluir aspectos afectivos que influencian el proceso cognitivo y se les conoce como agentes EBDI o Emotional BDI agents. Los agentes EBDI son agentes BDI extendidos para soportar el modelado afectivo. Los principales aspectos afectivos que se han modelado en los agentes EBDI son las emociones, el estado de ánimo y la personalidad. De estos el más desarrollado ha sido las emociones, ya que juegan un papel clave en el proceso cognitivo. A pesar del creciente desarrollo de los agentes EBDI, hay aspectos que todavia no se han considerado al modelar el afecto, como es el carácter disfuncional o funcional de las emociones (negativas o positivas), el cual es un termino terapéutico para clasificar la naturaleza de las emociones basado en el contenido de las creencias o cogniciones (irracional o racional) que les dió origen. Otro tema que ha sido escasamente tratado es la regulación de las emociones, y en el caso de la regulación del estado de ánimo, menos aún. Aunque muchas propuestas se han desarrollado buscando integrar la mayor cantidad posible de estos aspectos en el comportamiento de los agentes BDI, aún falta una propuesta que logre integrarlos todos de forma eficiente.

\section{El framework ABC-EBDI}

Nuestra propuesta es un framework para agentes EBDI que busca integrar la mayor cantidad posible de los aspectos y capacidades afectivas antes mencionadas. El framework ABC-EBDI, permitirá el modelado afectivo en cuanto a emociones, estado de ánimo y personalidad del agente, e integrará la 
regulación del afecto, teniendo en cuenta la influencia de cada uno en el proceso cognitivo BDI del agente. Para ellos modelaremos los aspectos afectivos a través del modelo ABC [8], que es un modelo terapéutico muy popular, en el que está basada la Terapia Relacional Emotivo Conductual (TREC), y no se ha usado hasta hoy en el modelado emocional de los agentes inteligentes. El modelo $\mathrm{ABC}$ permite lidiar con la clasificación de las emociones en cuanto a su naturaleza disfuncional y funcional $\mathrm{y}$, asi mismo, modelar la conducta mal-adaptativa y adaptativa, dependiendo en ambos casos del contenido irracional o racional de las cogniciones o creencias. En cuanto a el estado de ánimo y la personalidad será a través del modelo de los dos factores propuesto por [11] y el conocido modelo de personalidad OCEAN [9] respectivamente. En cuanto al modelado de las capacidades afectivas, modelaremos la regulación del afecto que incluye: la regulación emocional con el modelo de Gross [10] y regulación del estado de ánimo con el modelo de Larsen [11], este último escasamente visto en el modelado de los agentes inteligentes. Finalmente, será modelado el comportamiento del agente en cuanto a: gestos, expresiones faciales, postura corporal, voz, etc., através del modelo de Satir [12] que establece 5 patrones de comunicación universales usados por los individuos al expresar algo.

\section{Aplicación}

Para probar la viabilidad de nuestra propuesta se desarrollará un paciente virtual (PV) para el entrenaminto de los estudiantes de diferentes profesiones de la salud como la medicina, enfermería, trabajadores sociales, dietistas, y psicoteraputas en el ámbito de comunicación de mala noticias. El PV será un ECA que a través de los juegos de roles, interpretará un paciente con determinada personalidad, creencias y con un estado de ánimo inicial, el cual mediante el diálogo con el estudiante mostrá diferentes estados de ánimos, emociones, y conducta haciendo uso de los patrones de comunicación de Satir. El objetivo principal del sistema será el entrenamiento de los estudiantes proveyendo al profesor de las herramientas necesarias para evaluar el desempeño del estudiante, y así mismo servirá como herramienta de autoentrenamiento para este último.

\section{Conclusiones}

En este trabajo se ha propuesto el framework $\mathrm{ABC}$ EBDI para el modelado de los agentes inteligentes, el cual integra afecto en cuanto a emociones, estado de ánimo y personalidad, y la regulación del afecto atendiendo a regulación emocional y regulación del estado de ánimo. Como trabajo futuro tenemos el desarrollo del PV propuesto para el entrenamiento de los estudiantes de la salud en el ámbito de la comunicación de malas noticias, en aras de probar la viabilidad de nuestro framework.

\section{REFERENCIAS}

[1] J. Cassell, Embodied conversational agents. MIT Press, 2000.

[2] S. Kopp, L. Gesellensetter, N. C. Krämer, and I. Wachsmuth, "A Conversational Agent as Museum Guide - Design and Evaluation of a Real-World Application," in International Workshop on Intelligent Virtual Agents, 2005, pp. 329-343.

[3] S. Marsella, L. Johnson, and C. Labore, "Interactive pedagogical drama," in Proceedings of the 4th International Conference on Autonomous agents, 2000, pp. 301-308.

[4] P. Kenny and T. D. Parsons, "Intelligent Virtual Patients for Training Clinical Skills," J. Virtual Real. Broadcast., vol. 8, no. 3, 2011.

[5] M. Cavazza, F. Charles, and S. J. Mead, "Interacting with virtual characters in interactive storytelling," in In Proceedings of the first international joint conference on Autonomous agents and multiagent systems: part 1, 2002, pp. 318-325.

[6] C. Breazeal, "Emotion and sociable humanoid robots," Int. J. Hum. Comput. Stud., vol. 59, no. 1, pp. 119-155, 2003.

[7] A. S. Rao and M. P. Georgeff, "BDI Agents : From Theory to Practice," Proc. first Int. Conf. multiagent Syst., vol. 95, pp. 312-319, 1995.

[8] A. Ellis, Reason and emotion in psychotherapy. New York: Stuart, 1962.

[9] R. R. McCrae and O. P. John, "An introduction to the five-factor model and its applications.," J. Pers., vol. 60, no. 2, pp. 175-215, 1992.

[10] J. J. Gross, "The emerging field of emotion regulation: an integrative review.," Rev. Gen. Psychol., vol. 2, no. 5, pp. 271-299, 1998.

[11] R. J. Larsen, "Toward a science of mood regulation," Psychol. Inq., vol. 11, no. 3, pp. 129-141, 2000.

[12] S. Andreas and V. Satir, Virginia Satir, the patterns of her magic. Real People Press, 1991. 